\title{
Prevenção e tratamento da constipação intestinal da gestante: sob olhar de
}

\section{fisioterapeutas}

\author{
Prevention and treatment of constipation in pregnant women: under the eyes of physiotherapists \\ Prevención y tratamiento del estreñimiento en mujeres embarazadas: bajo la mirada de los
}

\section{fisioterapeutas}

Recebido: 22/10/2021 | Revisado: 31/10/2021 | Aceito: 05/11/2021 | Publicado: 08/11/2021

\author{
Ana Júlia Oliveira Cardoso \\ ORCID: https://orcid.org/0000-0001-9511-9642 \\ Faculdade Independente do Nordeste, Brasil \\ Email: anajuoliveirac@outlook.com \\ Juliana Braga Facchinetti Moura \\ ORCID: https://orcid.org/0000-0001-9621-801X \\ Faculdade Independente do Nordeste, Brasil \\ Email: julianafacchinetti@ fainor.com.br
}

\begin{abstract}
Resumo
Objetivo: Identificar as condutas adotadas por fisioterapeutas para prevenção e tratamento da constipação intestinal em gestantes. Metodologia: Trata-se de uma pesquisa de caráter descritivo e exploratório com abordagem qualitativa, que foi realizada de forma não presencial, em formato on-line. A população do estudo foi representada por fisioterapeutas atuantes na área de fisioterapia pélvica na cidade de Vitória da Conquista - BA. Foi utilizado como instrumento um questionário on-line elaborado pelas pesquisadoras na plataforma Google Forms, contendo perguntas para caracterizar o perfil sociodemográfico e ocupacional das participantes. Seguido de perguntas direcionadas ao tema em estudo onde os participantes puderam compartilhar por escrito, suas experiências e condutas adotadas na prevenção e tratamento de mulheres que possuem CI no período gestacional. Resultados: Responderam ao questionário 5 fisioterapeutas do sexo feminino, com idades entre 24 a 44 anos, onde a maioria possuía título de especialista na área de Fisioterapia Pélvica e relataram ter conhecimento e domínio de atuação da situação em estudo. As participantes compartilharam técnicas em comuns de prevenção e tratamento da CI em mulheres no período gravídico. Conclusão: Os resultados desta pesquisa demonstraram que as condutas utilizadas pelas fisioterapeutas aliadas ao envolvimento da equipe multidisciplinar são eficazes para prevenir e tratar a constipação intestinal em gestantes.
\end{abstract}

Palavras-chave: Constipação intestinal; Gravidez; Fisioterapia; Prevenção; Tratamento.

\begin{abstract}
Objective: To identify the approaches adopted by physical therapists for the prevention and treatment of intestinal constipation in pregnant women. Methodology: This is a descriptive and exploratory research with a qualitative approach, which was carried out in person, in an online format. The study population was represented by physical therapists working in the area of pelvic physical therapy in the city of Vitória da Conquista - BA. An online questionnaire developed by the researchers on the Google Forms platform was used as an instrument, containing questions to characterize the sociodemographic and occupational profile of the participants. Followed by questions directed to the topic under study where participants were able to share in writing, their experiences and behaviors adopted in the prevention and treatment of women who have IC during pregnancy. Results: Five female physiotherapists, aged 24 to 44 years old, answered the questionnaire, most of whom had a specialist title in the field of Pelvic Physiotherapy and reported having knowledge and mastery of the situation under study. Participants shared common techniques for the prevention and treatment of IC in pregnant women. Conclusion: The results of this research showed that the approaches used by physical therapists combined with the involvement of the multidisciplinary team are effective to prevent and treat constipation in pregnant women.
\end{abstract}

Keywords: Constipation; Pregnancy; Physiotherapy; Prevention; Treatment.

\section{Resumen}

Objetivo: Identificar los enfoques adoptados por los fisioterapeutas para la prevención y el tratamiento del estreñimiento intestinal en mujeres embarazadas. Metodología: Se trata de una investigación descriptiva y exploratoria con enfoque cualitativo, que se realizó de forma presencial, en formato online. La población de estudio estuvo representada por fisioterapeutas que trabajan en el área de fisioterapia pélvica en la ciudad de Vitória da 
Conquista - BA. Se utilizó como instrumento un cuestionario online desarrollado por los investigadores en la plataforma Google Forms, que contenía preguntas para caracterizar el perfil sociodemográfico y ocupacional de las participantes. Seguido de preguntas dirigidas al tema en estudio donde los participantes pudieron compartir por escrito, sus experiencias y comportamientos adoptados en la prevención y tratamiento de mujeres que tienen CI durante el embarazo. Resultados: Respondieron al cuestionario cinco fisioterapeutas, de 24 a 44 años, la mayoría con título de especialista en el campo de Fisioterapia Pélvica y refirieron tener conocimiento y dominio de la situación en estudio. Los participantes compartieron técnicas comunes para la prevención y el tratamiento de la CI en mujeres embarazadas. Conclusión: Los resultados de esta investigación demostraron que los enfoques utilizados por los fisioterapeutas combinados con la participación del equipo multidisciplinario son efectivos para prevenir y tratar el estreñimiento en mujeres embarazadas.

Palabras clave: Estreñimiento; El embarazo; Fisioterapia; Prevención; Tratamiento.

\section{Introdução}

A constipação intestinal (CI) é definida como passagem infrequente ou dificuldade de evacuação das fezes, estando ligada a sintomas como fezes duras, esforço, sensação de bloqueio anorretal, evacuação incompleta, desconforto abdominal e distensão abdominal (Jani \& Marsiano, 2018). A prevalência média de CI em adultos foi estimada em $16 \%$ em todo o mundo. No processo de envelhecimento essa porcentagem tende a aumentar, resultando em 33,5\% em adultos de 60 a 110 anos (Forootan; Bagheri \& Darvish, 2018).

Saffioti, et al., (2011) dizem que no Brasil são raros os estudos sobre prevalência de CI no período gestacional, apenas o trabalho de Kawaguti, et al., (2008) se destaca na literatura nacional, trazendo os seguintes dados: $24 \%$ das mulheres referem lentidão do trânsito intestinal, porém 19,5\% são efetivamente consideradas constipadas de acordo com os critérios de Roma, uma escala que tem como objetivo diagnosticar desordens gastrointestinais funcionais. No entanto, há a necessidade de realização de novos estudos que notifiquem de forma objetiva e padronizada a prevalência da CI na gestação.

De acordo com Forootan, Bagheri \& Darvishi (2018), a patogenia dessa disfunção é advinda de vários fatores, sendo eles a predisposição genética, nível socioeconômico, baixo consumo de fibras, falta de ingestão adequada de líquidos, falta de mobilidade, distúrbio no equilíbrio hormonal, efeitos colaterais de medicamentos ou anatomia do corpo. Durante a gravidez, as mudanças que ocorrem nos níveis hormonais e os efeitos físicos de um útero dilatado podem influenciar em um novo início de CI por trânsito lento ou agravar um quadro pré-existente (Wang, et al., 2020).

O aumento de progesterona no corpo, redução de exercícios e mais ingestão de proteína e gordura para atender às necessidades nutricionais durante a gravidez, faz com que mulheres grávidas venham apresentar taxas de prevalências mais altas (Shi, et al., 2015). Porém, segundo Saffioti, et al., (2011) são poucos os trabalhos que evoluem além da notificação dos sintomas e do período em que eles ocorrem.

A CI quando associada aos altos níveis de estresses provocados pelas alterações hormonais na mulher durante o período gestacional, agravam os problemas físicos, emocionais, e afetam significativamente a vida diária e o bem-estar da gestante. Sabe-se que a saúde geral, mental e função social em pessoas constipadas são inferiores às de indivíduos saudáveis, além de existir uma baixa procura por atendimento profissional a fim de resolver o problema, e assim, a maioria opta por automedicação (Forootan, et al., 2018). Porém, a terapia farmacológica pode ser insatisfatória, necessitando de uma avaliação detalhada em pacientes que apresentam essa disfunção, a fim de incluir medidas fisioterapêuticas no tratamento (Gotfried, 2021).

Nesse sentido, a fisioterapia pode ser uma opção para auxiliar e melhorar os sintomas das gestantes que tem CI, através das técnicas de treinamento para relaxar os músculos do assoalho pélvico, da coordenação neuromuscular, métodos de feedback visual, sonoro e verbal, além de outras terapias que promovem aumento da mobilidade gastrointestinal (Forootan, et al., 2018). 
Sendo assim, este estudo apresenta como objetivos identificar as condutas adotadas por fisioterapeutas para prevenção e tratamento da constipação intestinal em gestantes, caracterizar o perfil sociodemográfico e ocupacional dos fisioterapeutas e, identificar as condutas fisioterapêuticas que não foram eficazes para prevenção e/ou tratamento da constipação intestinal em gestantes.

\section{Metodologia}

Trata-se de uma pesquisa de caráter descritivo e exploratório com abordagem qualitativa. De acordo com Gerhardt \& Silveira (2009) diferente da pesquisa quantitativa, a pesquisa qualitativa além de mensurar um tema, descreve impressões, pontos de vistas e opiniões de um grupo social, não se preocupando com representatividade numérica.

Devido à pandemia e a situação do COVID-19, a fim de reduzir o contato, a pesquisa foi realizada em formato online, com fisioterapeutas atuantes na área de fisioterapia pélvica, na cidade de Vitória da Conquista - BA, Brasil.

As participantes atenderam aos seguintes critérios de inclusão: fisioterapeutas que trabalhavam na área da fisioterapia pélvica que atuam em obstetrícia na cidade de Vitória da Conquista - BA.

Foram excluídas da pesquisa os fisioterapeutas com menos de três anos de experiência na área de obstetrícia.

A coleta de dados foi iniciada somente após aprovação pelo Comitê de Ética e Pesquisa da Faculdade Independente do Nordeste (CEP/FAINOR), sob o parecer n 4.815.906. Foram respeitados os princípios éticos das resoluções n 466 de 2012 e n 510 de 2016 do Conselho Nacional de Saúde (CNS).

As pesquisadoras convidaram o público da pesquisa através do email encontrado no Google, e das redes sociais (whatsapp, instagram e facebook). Após aceitarem em participar da pesquisa, foi disponibilizado um link contendo o TCLE juntamente com o questionário on-line elaborado pelas pesquisadoras na plataforma Google Forms, contendo perguntas para caracterizar o perfil sociodemográfico e ocupacional das participantes. Seguido de perguntas direcionadas ao tema em estudo onde os participantes compartilharam por escrito, suas experiências e condutas adotadas na prevenção e tratamento de mulheres que possuem CI no período gestacional, explanando a eficácia das técnicas, bem como, condutas que não foram eficazes.

Os dados coletados foram analisados de acordo com o método de análise de conteúdo de Bardin, que são divididos em pré-análise, exploração do material e interpretação.

Bardin (2011) diz que a pré-análise ocorre quando as informações distribuídas no referencial teórico tem relação com o material coletado, indicando fatores comuns para interpretação dos mesmos. A exploração do material se baseia na separação de pontos relevantes, resumidos em parágrafos, dando sentido a tudo que foi apontado. E a interpretação é a compreensão dos conteúdos obtidos no questionário (Silva \& Fossa, 2015).

\section{Resultados e Discussão}

Os resultados foram divididos em dois blocos: primeiro, contendo a caracterização das participantes e, em seguida, as unidades temáticas.

Segundo Patton (2002), nenhum método simples resolve corretamente o problema em pesquisa e cada método revela diferentes aspectos da realidade empírica. Dessa forma, buscou-se triangular os resultados das análises, com a literatura existente e os dados das observações. 


\subsection{Caracterização das participantes}

Responderam ao questionário 5 fisioterapeutas do sexo feminino, com idades entre 24 a 44 anos, onde os nomes foram substituídos por pseudônimos para preservar a identidade. A maioria das participantes possuía título de especialista na área de Fisioterapia Pélvica e relataram ter conhecimento e domínio de atuação da situação em estudo. As participantes compartilharam técnicas em comuns de prevenção e tratamento da $\mathrm{CI}$ em mulheres no período gravídico.

De acordo com Santos, et al., (2017) a qualificação profissional é um conjunto de experiências posteriores à uma formação inicial, levando ao profissional uma manutenção, aumento ou melhora da sua competência, da qualidade dos serviços, com objetivo de melhorar a equidade no cuidado, tornando-os mais qualificados para o atendimento das necessidades da população.

\subsection{Condutas utilizadas para prevenir a constipação intestinal em gestantes}

Na visão das fisioterapeutas, é imprescindível que a atuação se inicie com a educação em saúde. "Descrevo como funciona o intestino, o que faz gerar a constipação." (Marcela). Além de explicar que a ação hormonal tem influencia nesta condição, mas que é necessário aprimorar a coordenação e percepção corporal.

Geniake, et al., (2015) apud Regra, Salerno \& Fernandes (2017) também considera a educação em saúde uma medida de intervenção, destacando a importância informar sobre os pontos da saúde materno-infantil, como além da anatomia e ações hormonais fisiológicas na gestação, a postura correta durante esse período, a percepção corporal e aleitamento materno após o nascimento da criança.

Logo em seguida, a atenção é com a percepção da mulher que está gestante em relação aos músculos do assoalho pélvico (MAP's), solicitando que perceba a respiração e sincronia com essa musculatura. “[...] existe uma sincronia entre os sistemas respiratório e dos MAP's, e se não existe percepção e coordenação, a contração não é efetiva." (Samanta).

Corroborando com Samanta, Kury (2019) apud Andrade (2021) diz que o assoalho pélvico é considerado o diafragma da região inferior, devido a sua conexão sinérgica e biomecânica com o sistema respiratório. Durante a inspiração e contração do diafragma, há um aumento de tensão gerando uma leve sobrecarga sobre o períneo, e para melhor estabilidade pélvica, é necessário que haja sincronia entre esses sistemas, associando a contração do assoalho pélvico com a respiração diafragmática.

Posteriormente a isso, é necessário que haja orientação quanto à posição de sentar no vaso sanitário, e se necessário acrescentar o uso da banqueta. "A finalidade desta orientação é fazer com que exista um relaxamento dos MAP's, e principalmente do músculo puboretal, para que a gestante não faça força para evacuar e sim, relaxe a musculatura." (Samanta).

Findlay, et al., (2010) apud Santinho (2019) enfatiza que a posição ao evacuar sentada, com uma ligeira flexão da parte superior do tronco e uso da banqueta, simulando a posição de cócoras, favorece o ângulo vesical correto, auxilia no trânsito intestinal, levando a uma frequência e consistência de fezes considerados dentro da normalidade, além de relaxar os MAP's, evitando assim, possíveis lesões anais.

As fisioterapeutas ainda ressaltam a liberação miofascial nas estruturas adjacentes e massagem em região perianal, com a finalidade de liberar tensões e facilitar o relaxamento e percepção dos MAP's.

De acordo com Biana, et al., (2021) a massagem perineal favorece o alongamento e aumenta a circulação da musculatura do AP, facilitando e melhorando a percepção, auto-conhecimento e função dessa região, além de promover integridade e proteção local durante a gestação e pós-parto.

Foi destacado os exercícios de mobilidade pélvica, que tem como finalidade fazer com que a gestante perceba e reconheça os músculos do assoalho pélvico. "Utilizamos a bola suiça, a própria ação da gravidade com a gestante em 
ortostase, e assim realizem os exercícios de mobilidade pélvica, associando à respiração e vocalização" (Samanta). Além disso, são priorizados exercícios na posição de cócoras, "[...] pois se trata da melhor posição fisiológica durante a evacuação." (Marcela).

A mobilidade pélvica possui resultados positivos sob o peristaltismo intestinal, e isso ocorre devido às repetições de exercícios e movimento, promovendo reeducação cognitiva, modificação, e percepção da musculatura abdominopélvica (Firmino, 2015 apud Jesus, Martins \& Fernandes, 2018).

Foi citada também a importância do trabalho multidisciplinar, o encaminhamento para profissionais que influenciam na prevenção desta disfunção. "encaminho a paciente para um profissional nutricionista a fim de auxiliar em uma dieta saudável e rica em fibras, muitas vezes encaminho ao psicólogo, pois a constipação pode acontecer devido problemas emocionais." (Marcela).

Segundo a Organização Mundial de Saúde, a assistência à gestante e puérpera deve ser multidisciplinar, multiprofissional e integral, levando consideração suas necessidades intelectuais, emocionais, sociais e culturais da mulher e de todos os envolvidos na sua rede apoio. Dessa forma, é possível ultrapassar os limites dos cuidados relacionados, apenas, aos sistemas biológicos (Sousa, et al., 2017).

\subsection{Condutas utilizadas e consideradas eficazes para tratar a constipação intestinal em mulheres no período gravídico}

Em complemento à questão anterior, as profissionais relataram que as condutas anteriores são eficazes para além de prevenir, tratar tal condição. E que além destas, a ativação dos músculos abdominais, o sentar em cima dos ísquios, o uso do balonete e o biofeedback são opções utilizadas nos consultórios para tratamento da CI na gestação. "Uso o balonete e realizo o treino expulsivo associado à respiração e relaxamento dos MAP's, e o biofeedback, no qual utilizo um dos programas de gráficos para visualização e treinamento com as gestantes." (Samanta).

O biofeedback é um aparelho bastante utilizado nas clínicas de fisioterapia pélvica, ele possibilita conscientização dos MAP através da utilização de um sinal sonoro e/ou visual, auxiliando assim na reabilitação da musculatura pélvica (Baracho, 2014 apud Nagamine, Dantas \& Silva, 2021).

O biofeedback manométrico é outra modalidade de tratamento, onde utiliza-se o balonete retal para favorecer a percepção do estiramento retal e a melhora da atividade esfincteriana em relação à coordenação muscular (Ferreira, 2018).

Foram citadas também, a Massagem de Vogler e termoterapia para auxiliar o bolo fecal na descida. Consolidando esse pensamento, Ferreira (2013) e Oliveira (2007) apud Jesus, Martins \& Fernandes (2018) dizem que a massagem auxilia no estímulo peristáltico e tem como vantagens o baixo custo, alto benefício e mínimos efeitos colaterais, sendo uma ótima opção para tratamento de CI. Além de causar um aumento da circulação local, como traz Felipe (2020) em seu estudo, que a termoterapia causa uma elevação da temperatura tecidual e fluxo sanguíneo local, aumentando o metabolismo e funcionamento intestinal.

Seguindo o mesmo raciocínio da questão anterior, foi destacada a importância da orientação em relação a pratica de atividade física e encaminhamento aos demais profissionais para tratamento multidisciplinar, como coloproctologista, nutricionista e psicólogo.

\subsection{Condutas ineficazes na prática clínica para a prevenção e/ou tratamento da constipação intestinal de gestantes}

Todas as participantes notaram que até o presente momento, obtiveram respostas positivas e eficazes com todas as técnicas utilizadas. Apesar de que há mulheres que se adaptam mais com algumas técnicas do que outras. "[...], pois como 
cada indivíduo é único e singular, a percepção e organização muscular e corporal perpassam o que é mais agradável, e melhor para o entendimento e realização dos exercícios preconizados.”, complementa Samanta.

Bruna diz que a melhora do quadro de cada paciente depende também da adesão das pacientes às orientações e mudanças nos hábitos diários.

\section{Conclusão}

Conclui-se, de acordo com os resultados obtidos, que as a educação e orientações em saúde, juntamente com as técnicas de massagem e liberação miofascial em região perineal, mobilidade pélvica, uso do balonete e biofeedback, massagem de vogler e termoterapia têm efeitos positivos nos sintomas da constipação intestinal em mulheres grávidas.

Além disso, os resultados desta pesquisa demonstraram que as boas práticas aliadas ao envolvimento da equipe multidisciplinar, podem fazer com que haja mudanças no padrão de tratamento no cenário obstétrico. Dessa forma, esta pesquisa pode vir a contribuir em outros estudos na área da fisioterapia pélvica em obstetrícia, a fim de qualificar o cuidado obstétrico, e para tanto, necessitam apropriar-se de referenciais que sustentem as práticas no campo obstétrico, bem como promover a rede de cuidados a fim de assegurar à mulher o direito à atenção humanizada não só na gravidez, como também no parto e no puerpério.

Uma das limitações deste trabalho foi à escassez de outros estudos abordando a temática, sendo assim sugere-se que novas pesquisas sejam feitas na área de fisioterapia pélvica em obstetrícia, com enfoque na prevenção e tratamento da constipação intestinal a fim de alcançar maior comprovação da eficácia das técnicas citadas e melhor observar os efeitos sistêmicos das mesmas.

\section{Referências}

Andrade, D. C. (2021). Importância do fortalecimento do assoalho pélvico em gestantes. Monografia (graduação). Repositório Universitário da Anima (RUNA). https://repositorio.animaeducacao.com.br/bitstream/ANIMA/13856/1/RUNA\%20De\%cc\%81borah\%20Coelho\%20de\%20Andrade\%20$\% 20$ Monografia\%20-\%20UniAGES.pdf.

Biana, C. B. et al. (2021). Terapias não farmacológicas aplicadas na gestação e no trabalho de parto: revisão integrativa. Revista da escola de enfermagem da USP, 55(03681). https://doi.org/10.1590/s1980-220x2019019703681.

Bradley, C. S. et al. (2007). Constipação na gravidez: prevalência, sintomas e fatores de risco. Obstetrícia e Ginecologia, $110(6)$, $1351-1357$. http://files.bvs.br/upload/S/0100-7254/2011/v39n3/a2502.pdf.

Burti, et al. (2006). Adaptações fisiológicas do período gestacional. Fisioterapia Brasil, 7(5), 375-380. https://portalatlanticaeditora.com.br/index.php/fisioterapiabrasil/article/view/1935.

Cestari et al. (2017). Análise das principais alterações posturais e sintomatologias decorrentes do período gestacional. Revista Ciência E Estudos Acadêmicos De Medicina, 1(08). https://periodicos.unemat.br/index.php/revistamedicina/article/view/1973.

Felipe, A. P. (2020). Atuação da fisioterapia na dor pélvica crônica feminina: uma revisão. Monografia (graduação). https://www.unirv.edu.br/conteudos/fckfiles/files/Andressa\%20Felipe\%20Pamplona.pdf.

Ferreira, J. B. (2018). Radiofrequência não ablativa perineal no tratamento da incontinência anal em mulheres: um estudo piloto. Monografia (mestrado). Repositório Institucional Escola Bahiana de Medicina e Saúde Pública. https://repositorio.bahiana.edu.br:8443/jspui/bitstream/bahiana/2199/1/DEFESA\%20CORRE\%c3\%87\%c3\%830-\%20\%20Juliana\%20Ferreira\%20\%283\%29-1.pdf.

Fonseca, M. L. V. (2016). Eletroestimulação transcutânea parassacral para bexiga hiperativa isolada em crianças e adolescentes. O papel da constipação. Monografia (doutorado). Repositório Institucional Escola Bahiana de Medicina e Saúde http://www.repositorio.bahiana.edu.br/jspui/bitstream/bahiana/224/1/Tese.\%20Fonseca.\%20Maria\%20Luiza\%20Veiga\%20da.\%202016.\%20001.\%20BAHIA NA.pdf.

Forootan, M., Bagheri, N., \& Darvishi, M. (2018). Constipação crônica: uma revisão de literatura. Medicina, 97(20), http://conicsemesp.org.br/anais/files/2019/1000003265.pdf.

Gotfried, J. (2020). Constipação. Manual MSD: Versão para profissionais de saúde. https://www.msdmanuals.com/pt-br/profissional/dist\%C3\%BArbiosgastrointestinais/sintomas-dos-dist\%C3\%BArbiosgastrointestinais/constipa\%C3\%A7\%C3\%A3o.

Gerhardt, E., \& Silveira, D. (2009). Métodos de pesquisa. Editora da UFRGS. 
Jani, B., \& Marsicano, E (2018). Constipação: Avaliação e Gestão. Missouri medicine, 115(3), 236-240. https://www.ncbi.nlm.nih.gov/pmc/art icles/PMC6140151/.

Jesus, D., Martins, T., Fernandes, I. (2018). Efeito da massagem abdominal e cinesioterapia em mulheres acometidas com constipação intestinal. Monografia (graduação). Uni América Centro Universitário. https://pleiade.uniamerica.br/index.php/bibliotecadigital/article/view/583/680.

Kawaguti, F., et al., Constipação na gravidez (2008). Rev bras. colo-proctol, 28(1), 46-49. https://www.scielo.br/j/rbc/a/Z3tbW8fJrJX8RJ xpVQQmkRP/?lang=pt.

Latorre, G., et al. (2020). Relação entre a falha no relaxamento do assoalho pélvico e a constipação em mulheres. $R$. bras. Ci. $e$ Mov, 28(1), 181-187. https://portalrevistas.ucb.br/index.php/RBCM/article/view/10252.

Luiz, D., Muniz, H., \& Almeida, M. (2017). Influência do exercício físico na qualidade de vida de gestantes. Journal of Development, 6(11), 86454-86469. https://www.brazilianjournals.com/index.php/BRJD/article/view/19603/15703.

Marins, A., Arzani, S., Lucas, T. (2008). Atividades terapêuticas para gestantes. Monografia (graduação). Repositório Institucional Faema https://repositorio.faema.edu.br/bitstream/123456789/140/1/ALMEIDA\%2c\%20L.\%20J.\%20C.\%20-\%20M\%c3\%89TODO\%20VODDER \%20COMO \%20 TR AT AMENTO $\% 20$ DE $\% 20$ EDEMA\%20GESTACIONAL\%20EFEITOS\%20DOS\%20RECURSOS\%20HIDROTER\%c3\%81PICOS\%20DURANTE\%2 $00 \%$ 20PER\%c3\%8dODO\%20GESTACIONAL..\%20REVIS\%c3\%830\%20BIBLIOGR\%c3\%81FICA.pdf

Nagamine, B., Dantas R.., Silva K. (2021). A importância do fortalecimento da musculatura do assoalho pélvico na saúde da mulher. Research, Society and Development, 10(2), 2525-3409. http://dx.doi.org/10.33448/rsd-v10i2.12894.

Pereira, J., Aguiar, V. (2016). Atividade Física e Gestação: Uma breve revisão de literatura. Monografia (graduação). Universidade Federal do Espírito Santo. https://cefd.ufes.br/sites/cefd.ufes.br/files/field/anexo/Jocilene\%20e\%20Victor\%20-\%20ATIVIDADE\%20F\%c3\%8dSICA\%20E\%20GESTA\%c3\%87\% c3\%83\% \% 20-\% 20UMA\%20BREVE\%20REVIS\%c3\%830\%20DE\%20LITERATURA.pdf.

Patton, M. Q. (2002). Qualitative research \& evaluation methods. Thousand Oaks, California: Sage Publications.

Regra, G., Salerno, G., \& Fernandes S. (2017). Educação em saúde para grávidas e puérperas. Revista Pesquisa em Fisioterapia. 7(3), 351-358. R https://www5.bahiana.edu.br/index.php/fisioterapia/article/view/1477.

Saffioti, R. F. et al. (2011). Constipação intestinal e gravidez. Femina, 39(3), 163-168. http://files.bvs.br/upload/S/0100-7254/2011/v39n3/a2502.pdf.

Santinho, M. J. C. (2019). Relatório de Estágio de Aprendizagem: Preparação para o Nascimento, Pós Parto e Disfunções do Pavimento Pélvico Proposta de Projeto de Implementação de Serviço de Fisioterapia em Saúde da Mulher. Monografia (relatório de estágio). Escola Superior de Saúde do Alcoitão. file:///C:/Users/tec/Downloads/Relat\%C3\%B3rio\%20Est\%C3\%A1gio\%20Final_MSnov19\%20(1).pdf.

Santini et al. (2019). Prevalência e fatores associados à ocorrência de incontinência urinária na gestação. Rev. Bras. Saúde Mater. Infant, 19(4), 975-982. https://www.scielo.br/j/rbsmi/a/s3TLFLSppFxgWmSVPGf9ydd/?lang=pt\&format=pdf.

Santos, M. C., Boechat Frauches, M. Rodrigues, S. M., \& Toledo Fernandes, E. (2017). Processo de Trabalho do Núcleo de Apoio à Saúde da Família (NASF): Importância da Qualificação Profissional. Saúde \& Transformação Social / Saúde e Mudança Social, 8 (2), 60-69. https://www.redalyc.org/pdf/2653/265352024007.pdf.

Saviani, D. (2009). Formação de professores: aspectos históricos e teóricos do problema no contexto brasileiro. Revista Brasileira de Educação, 14(40), 143155. https://www.scielo.br/j/rbedu/a/45rkkPghMMjMv3DBX3mTBHm/?format=pdf\&lang=pt.

Silva et al. (2018). Atuação do fisioterapeuta no período gestacional: uma revisão integrativa de literatura. ReonFacema. 4(4), 1330-1338. http://www.facema.edu.br/ojs/index.php/ReOnFacema/article/view/310.

Shi $\mathrm{W}$ et al (2015). Epidemiologia e fatores de risco de constipação funcional em mulheres grávidas. PloS one, 10 (7), e0133521. https://doi.org/10.1371/journal.pone.0133521.

Sousa, V. P. S. (2017). Percepção das participantes de um curso para gestantes sobre a abordagem multidisciplinar em saúde. Revista Pesquisa Em Fisioterapia, 7(1), 79-86. https://doi.org/10.17267/2238-2704rpf.v7i1.1261.

Tamura, et al. (2016). Prevalência e auto-conhecimento da constipação crônica: resultados de uma pesquisa na internet. Journal of Neurogastroenterology and Motility. 22(4), 677-685. https://www.jnmjournal.org/journal/view.html?doi=10.5056/jnm15187.

Wang, M. D. et al. (2020). Tratamento do íleo induzido por constipação de trânsito lento durante a gravidez por colectomia com anastomose ileorretal. Medicina, 28(2), 241-245. https://www.scielo.br/j/rbc/a/MF8vDfsKfrQvZLDWmY9ywgC/?lang=pt\&format=pdf. 